\title{
CORRESPONDENCE
}

\section{Italy leads the way in supporting African biotechnology}

SIR - In your News story

'African science drops down G8

agenda' (Nature 460, 16; 2009),

you express concern about the

lack of interest that has been

shown by the $\mathrm{G} 8$ countries in

the development of science in

Africa. But there are bright spots:

for example, a laboratory of

international standard recently

opened in South Africa as a direct result of G8 discussions, with Italy

taking the lead towards its rapid realization.

In the final declaration of the 2005 G8 summit in Gleneagles, heads of state and government considered the possibility of establishing an African branch of the International Centre for Genetic Engineering and Biotechnology (ICGEB, www. icgeb.org) to do research into diseases afflicting the continent. Action followed promptly, and the new ICGEB component was inaugurated in Cape Town in September 2007, joining others in Trieste and Delhi. In less than two years, the difficulties inherent in establishing a new research institution were overcome; these included meeting complex legal requirements as a result of the intergovernmental nature of our organization.

This achievement was possible because of generous funding and support by the South African and Italian governments. Today, more than 50 scientists are working in the new laboratories, producing high-level biomedical science, sparking collaboration with other research institutes in Africa and training young scientists from the whole continent.

ICGEB is also implementing an important project from Cape Town, which is funded by the Bill and Melinda Gates Foundation and aimed at building technical and scientific capabilities in sub-Saharan Africa for the effective regulation of biotechnology in agriculture.
Although ICGEB activities in

Africa are still limited, they are actually happening. Regrettably, these activities are not as adequately noticed as they should be, perhaps because of a preference for highlighting projects rather than facts.

Francisco E. Baralle International Centre for Genetic Engineering and Biotechnology, AREA Science Park, Padriciano 99, 34149 Trieste, Italy e-mail: baralle@icgeb.org

\section{Investors likely to venture back as crisis subsides}

\section{SIR - As an investor and an} independent adviser to corporate management, I am not convinced by John Browning's arguments that venture funding is unlikely to bounce back, as suggested by his Essay 'The incredible shrinking venture capital' (Nature 460, 459; 2009).

Earlier trends indicate that venture-capital funding will probably rebound after the current financial crisis subsides. Until last year, venture-capital investments in US companies had increased annually for 12 years, except during the dot-com bubble of 1999-2001.

The biotechnology sector, in particular, reported a substantial positive trend in these investments during the same period: their annual funding grew even when quarterly funding fell for one or two quarters by more than $30 \%$ relative to the previous quarter.

Meanwhile, most of the technologies that are crucial to solving societal problems drugs, solar energy, biofuels and nanotechnology - will address huge markets and require large amounts of high-risk capital. Those that succeed will either be acquired or be taken public.

For every ten investments that a venture-capital firm makes, it needs only one or two big winners to offset the three or four investments that fail and the remainder that seem to go nowhere.

J. Leslie Glick 12306 Ashville Drive, Tampa, Florida 33626, USA e-mail: jlglick@ix.netcom.com

\section{Czech bibliometric system fosters mediocre research}

SIR - Following your News story

'Czech researchers angry over government changes' (Nature 460,$157 ; 2009$ ), we would like to point out that ill-conceived bibliometrics can be used as an excuse for deep cuts in basic research funding and the diversion of funds to 'applied' research.

The innovative evaluation system endorsed by the Czech government's research and development council ascribes a certain number of points to every paper, patent, technology and piece of software. However, it does not properly take into account the originality of the result or the publication medium, nor does it consider the different financial requirements of different branches of science.

This system effectively penalizes inter-institutional cooperation and promotes inflation of mediocre science. It discredits the whole concept of evaluation of science based on scientiometrics.

It does not soothe our pain that publication of this letter will confer as many points on our institutions as a paper in Progress in Polymer Science or three in The American Bankruptcy Law Journal. Jan Konvalinka, Helena Illnerová, Pavel Hobza, Václav Hořejší, Antonín Holý, Pavel Jungwirth, Václav Pačes Academy of Sciences of the Czech Republic

e-mail: konval@uochb.cas.cz

Pavel Martásek First Faculty of

Medicine, Charles University,

Prague, Czech Republic

Jiři Zlatuška Faculty of Informatics,

Masaryk University, Brno,

Czech Republic

\section{Non-scientists could still contribute to reform of the ERC}

SIR - The mid-term review of the European Research Council (ERC), the European homologue of the US National Science Foundation, draws attention to some of the council's bureaucratic shortcomings.

Towards a World-class Frontier Research Organisation (http:// tinyurl.com/noa7ra), published in July, highlights the ERC's initial successes. But it points out that the council's long-term sustainability depends on a drastic reduction in red tape, better governance structures, more autonomy under a bettersuited legal framework and several other reforms.

The reviewers argue that ERC steering bodies should not include too many non-scientists, nor be run by them. But an over-enthusiastic exclusion of non-scientists risks conveying a 'closed shop' impression. It would fly in the face of the principle of diversity management, in which different competencies and backgrounds are deployed to run a complex, multi-faceted societal enterprise such as the ERC.

Such a scheme would also undermine the professionalization that is proclaimed in the report, as well as closing doors to the taxpayer and people outside the inner rank and file. We must presume that this is not the reviewers' intention, but the point nevertheless calls for clarification, not least to enhance the impact of an otherwise strong report.

Wilhelm Krull VolkswagenStiftung, Kastanienallee 35, 30519 Hanover, Germany e-mail: krull@volkswagenstiftung.de Jakob Edler Manchester Institute of Innovation Research, University of Manchester, Manchester M13 9PL, UK Michael Stampfer Vienna Science and Technology Fund, Währinger Strasse 3/15a, 1090 Vienna, Austria

Contributions may be submitted tocorrespondence@nature.com. 